\title{
A flexible cable-shaped supercapacitor based on carbon fibers coated with graphene flakes for wearable electronic applications
}

\author{
Jiyoung Kim ${ }^{1}$, Junli Yin ${ }^{1,2}$, Xing Xuan ${ }^{1}$ and Jae Yeong Park ${ }^{*}$ (D)
}

\begin{abstract}
This work presents a flexible cable-shaped supercapacitor based on carbon fibers (CFs) coated with graphene flakes (GFs) for wearable electronic applications. The CF bundles were adopted as base materials and the GFs were coated on the surface of CFs using a simple dipping method for the enhancement of the specific surface area and the higher conductivity of flexible electrodes. $\mathrm{H}_{2} \mathrm{SO}_{4}$ was mixed with poly(vinyl alcohol) (PVA) to form a gel electrolyte, which can prevent leakage. Polydimethylsiloxane (PDMS) was selected as a packaging material to fabricate the proposed flexible supercapacitor due to its flexibility and good thermal and chemical stability. From the electrochemical analysis, the fabricated device exhibited $15.099-6.492 \mathrm{mF} / \mathrm{cm}^{2}$ of specific capacitance and $2.097-0.902 \mu \mathrm{Wh} / \mathrm{cm}^{2}$ of energy density in the range of 50-300 mV/s of scan rate. These values were about 1.9 times larger than the supercapacitor without being coated with the GFs. In addition, the specific capacitance showed small difference of $3.4 \%$ between straight and twisted positions, which assures the mechanical stability of the flexible cable-shaped supercapacitor.
\end{abstract}

Keywords: Flexible supercapacitor, Carbon fibers, Graphene flakes, Gel electrolyte, Wearable electronics

\section{Introduction}

As wearable electronic devices become popular, there is an increasing demand for flexible energy storage devices which have a large output range and power density [1-2]. Furthermore, energy storage devices should be flexible, lightweight and compatible with wearable electronic devices. Therefore, various types of energy storage devices such as supercapacitors [3], solar cells [4], lithium-ion batteries [5] and thermoelectric generators [6] have been researched over the last decade. Among those developed devices, supercapacitors, also called electrochemical capacitors or ultracapacitors, have been considered as the most adequate alternative for wearable electronic applications through their attractive features such as long lifecycle and high power density [7]. In addition, they have several advantages like a wide temperature range and chemical stability, which are appropriate

\footnotetext{
*Correspondence: jaepark@kw.ac.kr

${ }^{1}$ Department of Electronic Engineering, Kwangwoon University, Seoul 01897, Republic of Korea

Full list of author information is available at the end of the article
}

for the wearable purpose. For example, Some groups presented supercapacitors based on flexible substrate using photolithography process [8-11]. Meng et al. reported a polymer-based thin supercapacitor with carbon nanotube (CNT)/polyaniline (PANI) nanocomposite electrodes using an electropolymerization method [12]. Some other groups presented printed planar supercapacitor using different printing method [13-16]. However, in these works, the deposited electrode materials were exposed, which can easily be detached from their substrates and nonelastic substrates were applied, which limit their use in wearable electronic applications [17-18].

In this research, to meet the demands of flexibility, we applied a polydimethylsiloxane (PDMS) elastomer due to its simple fabrication process and low Young's modulus of $1.8 \mathrm{MPa}$, which also shows an elongation at a failure of $160 \%$, a material with chemical and thermal stability. For example, Chen et al. fabricated a stretchable supercapacitor based on carbon nanotube sheets on the PDMS [19]. However, it showed a limitation for wearable electronic applications since the device was not fully packaged. 
Therefore, the exposed gel electrolyte can cause performance degradation and its acidic feature is not biocompatible to human skin. Also, it has the problem of mechanical stability under repeated deformations such as bending and stretching.

In this research, we successfully developed a flexible cable-shaped supercapacitor based on carbon fibers (CFs) coated with graphene flakes (GFs) for wearable electronic applications. For fabricating electrodes, CF bundles were adopted as base materials. For the enhancement of the specific surface area and conductivity of the electrodes, GFs were coated on the CFs using a dipping method. For the electrolyte, $\mathrm{H}_{2} \mathrm{SO}_{4}$ was mixed with poly(vinyl alcohol) (PVA) for the formation of the gel phase, which makes it free from leakage problem. Lastly, for packaging all these materials, PDMS was selected owing to its flexibility and good thermal and chemical stability, also maintains the proposed supercapacitor's electrochemical properties under mechanical deformation.

\section{Design and fabrication}

The proposed flexible cable-shaped supercapacitor consists of electrodes, gel electrolyte, and PDMS elastomer, which was used for packaging the device as shown in Fig. 1. For the electrodes, CFs $(7 \mu \mathrm{m}$ diameter, TORAYCA ${ }^{\circledR}$, Toray Industries, Inc., Japan) were selected as base materials. These CFs have good electrical and mechanical properties with long shape, which is appropriate for the cable-shaped supercapacitor [20]. For the increment of specific surface area and electric properties of the electrodes, GFs were deposited on the surface of fiber bundle by dip-coating into graphene dispersion $(7 \mathrm{~nm}$ average thickness, Graphene Supermarket, U.S.A.) diluted with $n$-butyl acetate (Supelco Inc.,

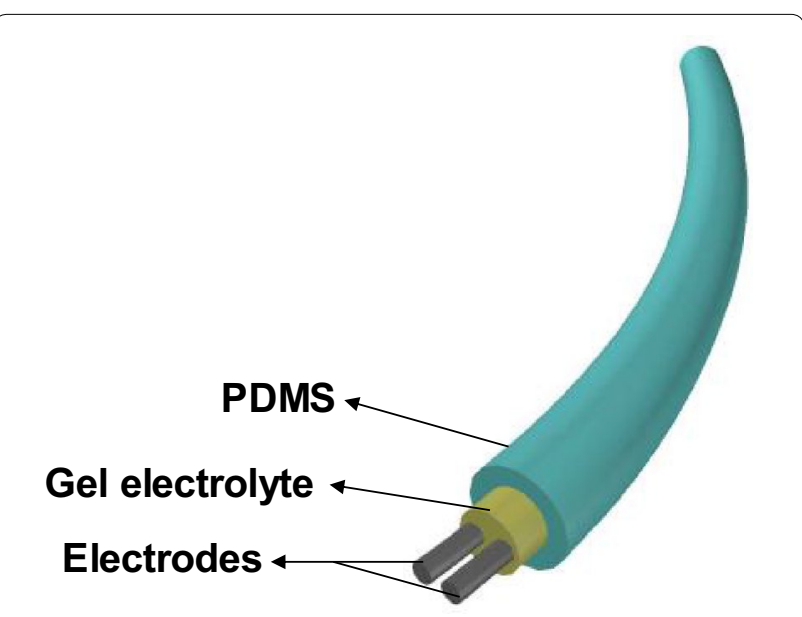

Fig. 1 Schematic diagram of the proposed flexible cable-shaped supercapacitor
U.S.A.). For the electrolyte, $\mathrm{H}_{2} \mathrm{SO}_{4}$ (ACS reagent, 95.098.0\%, Sigma-Aldrich, U.S.A.) was selected because of its high capacitance among other materials which were used for supercapacitors in previous research [21]. Thus, in order to form a gel phase, PVA $\left(M_{w} 89,000-98,000\right.$, $99+\%$ hydrolyzed, Sigma-Aldrich, U.S.A.) was mixed and heated with $\mathrm{H}_{2} \mathrm{SO}_{4}$. Finally, PDMS (Sylgard ${ }^{\circledR} 184$, Dow Corning) was selected as the packaging material due to its novel chemical and thermal stability [22].

The schematic diagram of fabrication sequences is shown in Fig. 2. First, two bundles of $20 \mathrm{~cm}$ CFs were dip-coated in the $7 \mathrm{wt} \%$ of graphene dispersion diluted with $n$-butyl acetate (Sigma-Aldrich, U.S.A.) and heated at $250{ }^{\circ} \mathrm{C}$ for $150 \mathrm{~min}$ to evaporate the solvent. GFscoated CF bundles were then twisted with a wire rotator (Lab Mart, South Korea) 20 times in order to form each electrode. Gel electrolyte was fabricated in the following steps: $100 \mathrm{~mL}$ deionized (DI) water, $10 \mathrm{~mL} \mathrm{H}_{2} \mathrm{SO}_{4}$ (ACS reagent, 95.0-98.0\%, Sigma-Aldrich, U.S.A.) and $10 \mathrm{~g}$ PVA were stirred and heated on a hot plate stirrer at $180{ }^{\circ} \mathrm{C}$ and $600 \mathrm{rpm}$ for $40 \mathrm{~min}$. The electrolyte solution became transparent after $20 \mathrm{~min}$ and turned into a gel phase when the solution temperature reached $70{ }^{\circ} \mathrm{C}$. After disposing of a pair of CF bundles, the pre-made gel electrolyte was coated and dried at room temperature for $24 \mathrm{~h}$. To make a cable shaped device, a commercial silicone shrinkable tube was employed as a mold for the packaging material, PDMS. Before placing the CF bundles, the release agent was sprayed inside the tube for easy removal of the tube after curing the PDMS. After placing the bundles into the tube, PDMS solution mixed the base and curing agent at the ratio of 20:1 was injected using a syringe. To prevent changing the electrolyte properties, the PDMS was cured at room temperature for $48 \mathrm{~h}$ and the tube was finally detached from the PDMS. The proposed flexible cable-shaped supercapacitor was successfully fabricated with $20 \mathrm{~cm}$ in length and $4 \mathrm{~mm}$ in thickness. For the electrochemical measurements, commercial copper wires were connected to both ends of the electrodes of supercapacitor with Ag paste.

\section{Experimental results and discussion}

Figure 3a shows a photograph of the fabricated flexible cable-shaped supercapacitor. As mentioned in the design and fabrication section, the $\mathrm{H}_{2} \mathrm{SO}_{4} / \mathrm{PVA}$ gel electrolytecoated CFs/GFs electrodes were surrounded by PDMS providing highly flexible structure. The fabricated supercapacitor could be changed into various states like freely bent-state (Fig. 3b) and twisted-state (Fig. 3c) and with its high flexibility. It was found that the electrodes and the electrolyte were fully embedded into the PDMS in stable position. 

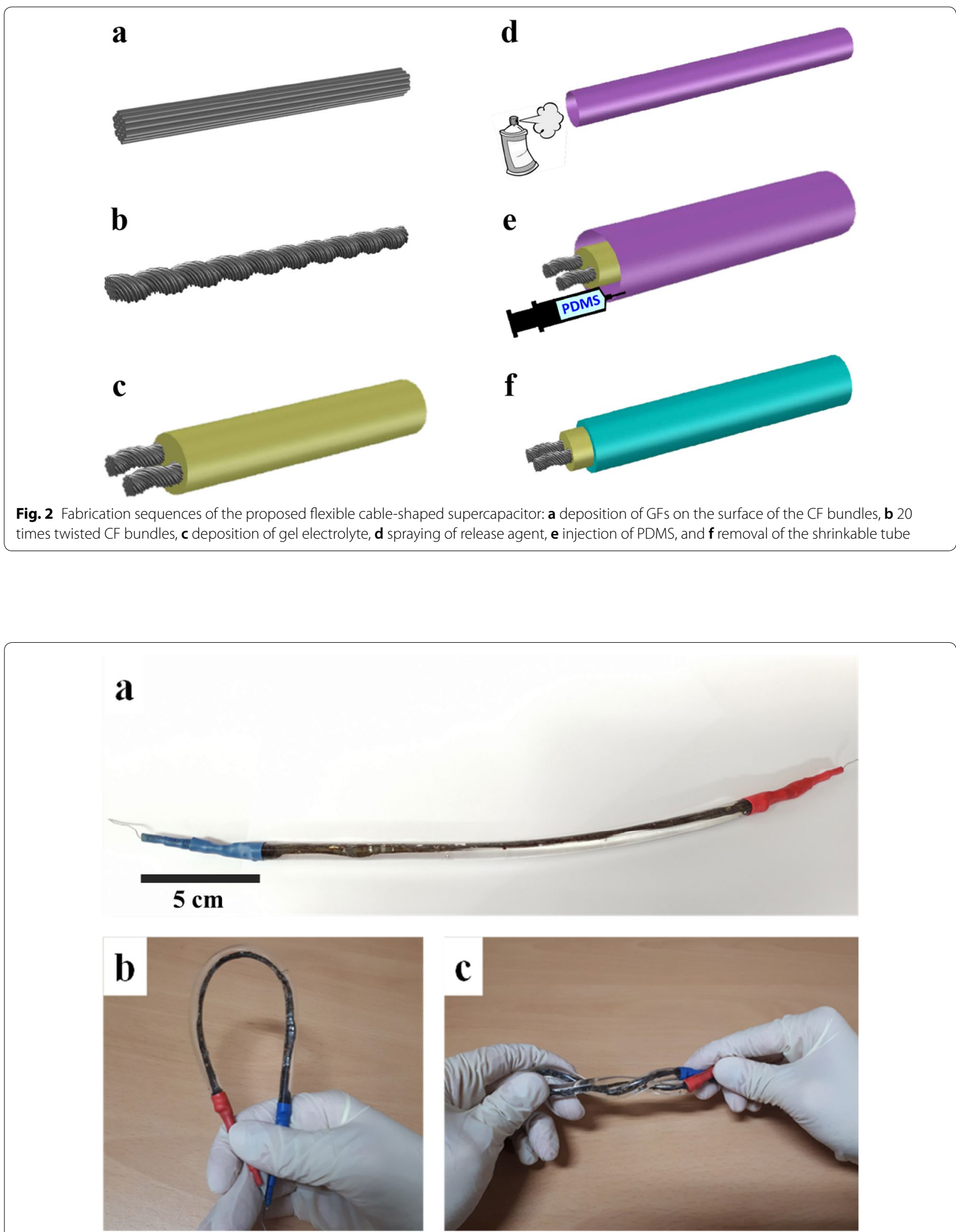

Fig. 3 Photographs of the fabricated flexible cable-shaped supercapacitor: $\mathbf{a}$ normal state, $\mathbf{b}$ bent-state, and $\mathbf{c}$ twisted-state 

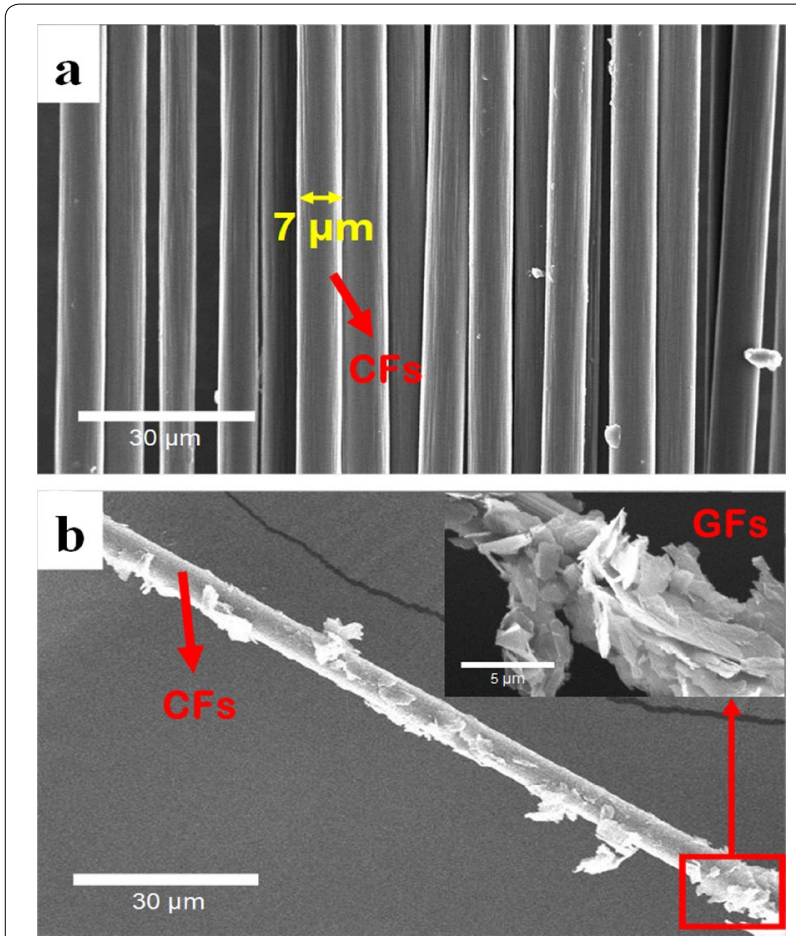

Fig. 4 FE-SEM images: $\mathbf{a}$ before and $\mathbf{b}$ after being coated with GFs on the surface of CFs

The surface morphologies of the bare and GFs-coated CFs were analyzed using a field emission scanning electron microscopy (FE-SEM) (S-4800, Hitachi High-Technologies Co., Ltd.) operating at $10 \mathrm{kV}$. It can be observed that the bare CFs show clear and planar surface as shown in Fig. 4a. However, after the deposition of GFs, the rougher surface as shown in Fig. 4b was observed, which attributes to higher active surface area and specific capacitance.

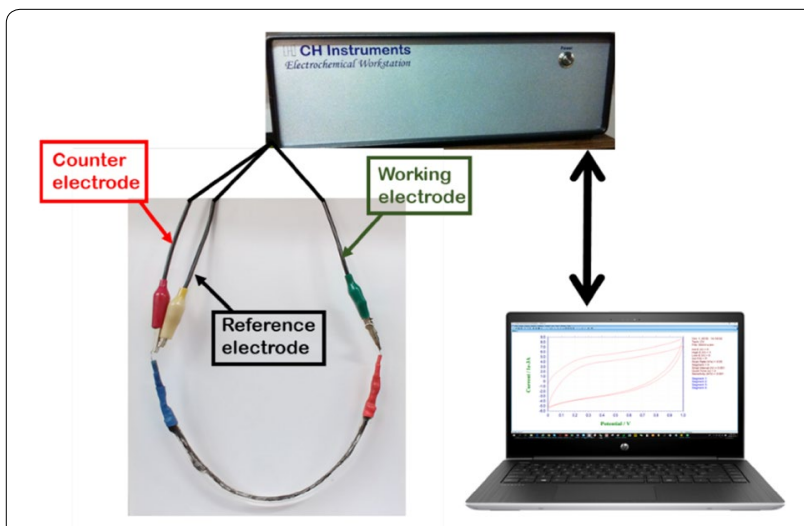

Fig. 5 Test setup for the electrochemical analysis of the fabricated supercapacitor
Finally, for the electrochemical characterization of the fabricated flexible supercapacitor, cyclic voltammetry $(\mathrm{CV})$ and galvanostatic charging-discharging (GCD) measurements were performed using an electrochemical workstation (660E, CH Instruments, U.S.A.) in straight and twisted states. Test setup was depicted in Fig. 5. The $\mathrm{CV}$ curves measured in a potential range from 0 to $1 \mathrm{~V}$ at
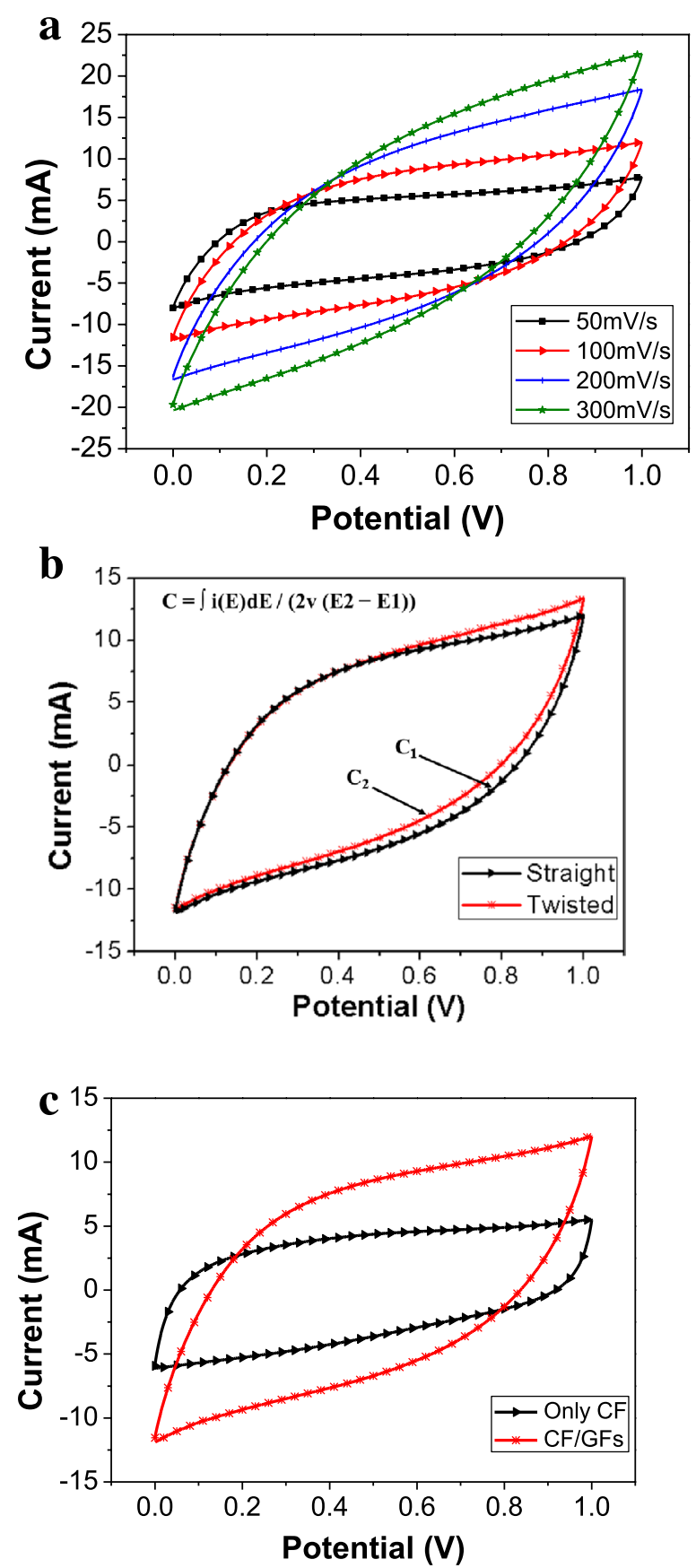

Fig. 6 Cyclic voltammetry (CV) curves: a under various scan rates, comparison between $\mathbf{b}$ straight and twisted position, and $\mathbf{c}$ before and after being coated with GFs at the scan rate of $100 \mathrm{mV} / \mathrm{s}$ 


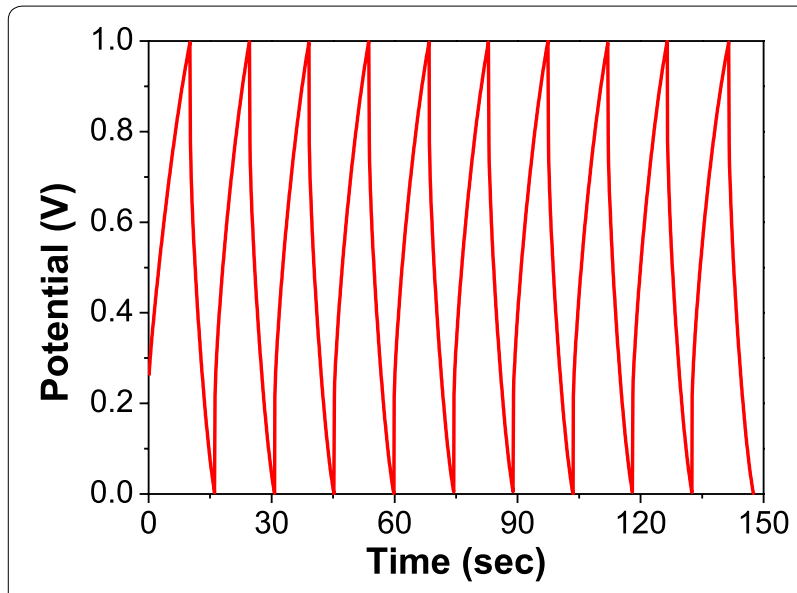

Fig. 7 Galvanostatic charging-discharging (GCD) curve under current of $10 \mathrm{~mA}$ various scan rates from 50 to $300 \mathrm{mV} / \mathrm{s}$ were presented in Fig. 6a. It can be observed that the CV curves show a rectangular shape, which means that the cable-shaped supercapacitor shows an electric double-layer capacitor (EDLC) behaviors. The comparison between CV curves at the straight and twisted position was shown in Fig. 6b, which showed $3.4 \%$ of small difference in specific capacitance (calculated form mentioned equation in the figure). Figure $6 \mathrm{c}$ indicates the comparison between before and after the coating of GFs on the CF surface. After the GFs coating, the integrated area of the CV curve was about 1.9 times larger than that of before coating, this value is proportional to specific capacitance and energy density. In addition, the comparison between $\mathrm{CV}$ curves at the normal and under stress position was shown in Fig. 6b. It indicated that performances of our fabricated

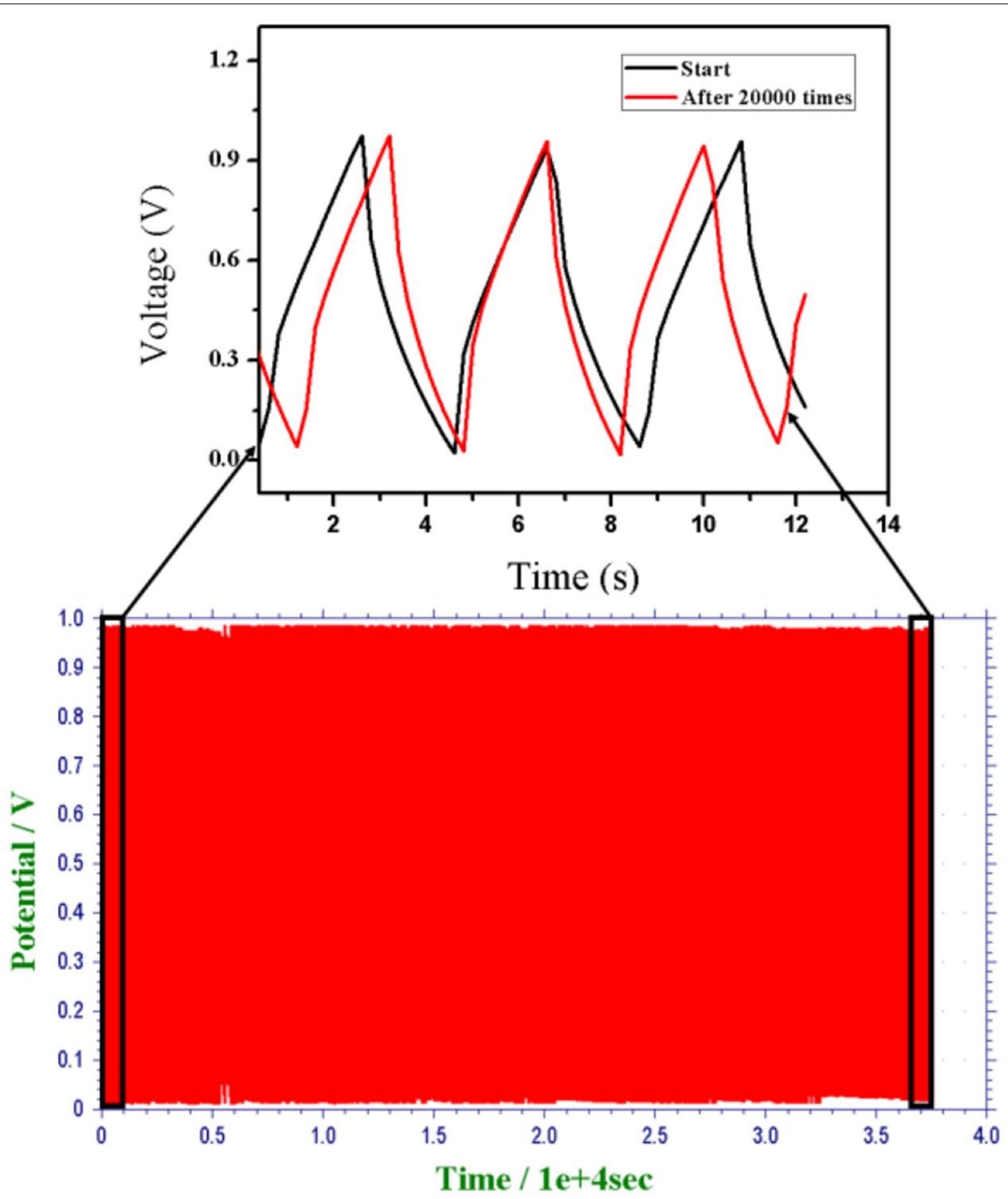

Fig. 8 Galvanostatic charging-discharging curves and its cycle life (20,000 segments) 
Table 1 Comparison of the capacitance, energy density of our supercapacitor with other works

\begin{tabular}{|c|c|c|c|c|c|c|}
\hline Electrode material & Preparation method & Substrate & Electrolyte & $C_{A}\left(\mathrm{mF} \mathrm{cm}^{-2}\right)$ & $E_{A}\left(\mu W h \mathrm{~cm}^{-2}\right)$ & References \\
\hline rGO & $\begin{array}{l}\text { Photolithography and in-situ } \\
\text { assembled graphene }\end{array}$ & PET & $\mathrm{PVA}-\mathrm{H}_{2} \mathrm{SO}_{4}$ & 0.95 & - & [23] \\
\hline LSG & Laser-scribed graphene & Flexible substrate & $\mathrm{PVA}-\mathrm{H}_{2} \mathrm{SO}_{4}$ & 2.32 & 0.32 & [24] \\
\hline EG/PH1000 & Spray coating & Paper/PET & $\mathrm{PVA}-\mathrm{H}_{2} \mathrm{SO}_{4}$ & 5.4 & - & {$[25]$} \\
\hline GFs-CFs & GFs coating & PDMS & $\mathrm{PVA}-\mathrm{H}_{2} \mathrm{SO}_{4}$ & 15.099 & 2.097 & This work \\
\hline
\end{tabular}

supercapacitor will not change much even under different situations. At last, the GCD measurement was analyzed and shown in Figs. 7 and 8. they showed quite uniform charging and discharging time to indicate the good energy storage performance and good life cycle of the fabricated cable-shaped supercapacitor. From CV and GCD measurements, $15.099-6.492 \mathrm{mF} / \mathrm{cm}^{2}$ of specific capacitance and $2.097-0.902 \mu \mathrm{Wh} / \mathrm{cm}^{2}$ of energy density were obtained in the range of $50-300 \mathrm{mV} / \mathrm{s}$ of scan rate, respectively. The comparison between our fabricated supercapacitor and other works was shown in Table 1 and it shows good performances in the wearable supercapacitor devices.

\section{Conclusions}

In summary, this research reports a flexible cableshaped supercapacitor based on CFs coated with GFs for wearable electronic applications. The graphenecoated flakes on the surface of CFs much improved the electrodes' specific surface area and electrical conductivity, which leads to higher specific capacitance. From the electrochemical analysis, the obtained rectangular shape of the CV curves showed the ideal EDLC property of the flexible cable-shaped supercapacitor. The high specific capacitance of 15.099-6.492 $\mathrm{mF} / \mathrm{cm}^{2}$ and energy density of $2.097-0.902 \mu \mathrm{Wh} / \mathrm{cm}^{2}$ were obtained in the range of $50-300 \mathrm{mV} / \mathrm{s}$ of scan rate, respectively. These values were about 1.9 times larger than the supercapacitor without being coated with GFs on the surface of CFs. Also, the specific capacitance just showed $3.4 \%$ of difference between straight and twisted position, which assures the mechanical stability of the fabricated flexible cable-shaped supercapacitor.

\section{Authors' contributions}

JK designed fabrication and experiments, and prepared the manuscript of this study. JY and XX participated in the analysis of this study. JYP conceived of the study, and participated in its design and coordination and helped to draft the manuscript. All authors read and approved the final manuscript.

\section{Author details}

${ }^{1}$ Department of Electronic Engineering, Kwangwoon University, Seoul 01897, Republic of Korea. ${ }^{2}$ School of Electrical Engineering, University of South China, Hengyang 421001, Hunan, People's Republic of China.
Acknowledgements

Not applicable.

\section{Competing interests}

The authors declare that they have no competing interests.

\section{Availability of data and materials}

All data generated or analysed during this study are included in this published article.

\section{Funding}

This work was supported by the Technology Innovation Program (20000773, Development of nanomultisensors based on wearable patch for nonhematological monitoring of metabolic syndrome) funded by the Ministry of Trade, Industry \& Energy (MI, Korea).

\section{Publisher's Note}

Springer Nature remains neutral with regard to jurisdictional claims in published maps and institutional affiliations.

Received: 3 January 2019 Accepted: 20 April 2019

Published online: 23 April 2019

References

1. Jost K, Stenger D, Perez CR, McDonough JK, Lian K, Gogotsi Y, Dion G (2013) Knitted and screen printed carbon-fiber supercapacitors for applications in wearable electronics. Energy Environ Sci 6:2698-2705

2. Pasta M, La Mantia F, Hu L, Deshazer HD, Cui Y (2010) Aqueous supercapacitors on conductive cotton. Nano Res 3:452-458

3. Xiao X, LiT, Yang P, Gao Y, Jin H, Ni W, Zhan W, Zhang X, Cao Y, Zhong J, Gong L, Yen WC, Mai W, Chen J, Huo K, Chueh YL, Wang JL, Zhou J (2012) Fiber-based all-solid-state flexible supercapacitors for self-powered systems. ACS Nano 6(10):9200-9206

4. Pan S, Yang Z, Chen P, Deng J, Li H, Peng H (2014) Wearable solar cells by stacking textile electrodes. Angew Chem 126:6224-6228

5. Hoshide T, Zheng Y, Hou J, Wang Z, Li Q, Zhao Z, Ma R, Sakaki T, Geng F (2017) Flexible lithium-ion fiber battery by the regular stacking of two-dimensional titanium oxide nanosheets hybridized with reduced graphene oxide. Nano Lett 17(6):3543-3549

6. Francioso L, De Pascali C, Farella I, Martucci C, Creti P, Siciliano P, Perrone A (2011) Flexible thermoelectric generator for ambient assisted living wearable biometric sensors. J Power Sources 196:3239-3243

7. Li X, Wei B (2013) Supercapacitors based on nanostructured carbon. Nano Energy 2:159-173

8. Si W, Yan C, Chen Y, Oswald S, Han L, Schmidt OG (2013) On chip, all solidstate and flexible micro-supercapacitors with high performance based on MnOx/Au multilayers. Energy Environ Sci 6:3218-3223

9. Huang P, Heon M, Pech D, Brunet M, Taberna PL, Gogotsi Y, Lofland S, Hettinger JD, Simon P (2013) Micro-supercapacitors from carbide derived carbon (CDC) films on silicon chips. J Power Sources 225:240-244

10. Majumdar R, Foroutan V, Paprotny I (2015) Post-release stressengineering of surface-micromachined MEMS structures using evaporated chromium and in-situ fabricated reconfigurable shadow masks. In: Proceedings of 
the 28th IEEE international conference micro electro mechanical systems (MEMS), pp 296-299

11. Majumdar R, Paprotny I (2017) Lithography-free self-reconfigurable postrelease stress engineering of surface micro-machined MEMS structures. J Micro Electro Mech Syst 26:671-678

12. Meng C, Liu C, Chen L, Hu C, Fan S (2010) Highly flexible and all-solidstate paperlike polymer supercapacitors. Nano Lett 10:4025-4031

13. Chang Q, Li L, Sai L, Shi W, Huang L (2018) Water-soluble hybrid graphene ink for gravure-printed planar supercapacitors. Adv Electron Mater 4(8):1800059

14. Tanwilaisiri A, Xu Y, Zhang R, Harrison D, Fyson J, Areir M (2018) Design and fabrication of modular supercapacitors using 3D printing. J Energy Storage 16:1-7

15. Hussain SA, Ward S, Mahdavipour O, Majumdar R, Paprotny I (2015) Untethered microscale flight: mechanisms and platforms for future MEMS aerial microrobotics. In: SPIE sensing technology + applications, 94940F-12

16. Ward S, Foroutan V, Majumdar R, Mahdavipour O, Hussain SA, Paprotny I (2015) Towards microscale flight: fabrication, stability analysis, and initial flight experiments for $300 \times 300 \times 1.5$ sized untethered MEMS microfliers. IEEE Trans Nanobiosci 14:323-331

17. Peng Z, Ye R, Mann JA, Zakhidov D, Li Y, Smalley PR, Lin J, Tour JM (2015) Flexible boron-doped laser-induced graphene microsupercapacitors. ACS Nano 9(6):5868-5875

18. Feng JX, Ye SH, Lu XF, Tong YX, Li GR (2015) Asymmetric paper supercapacitor based on amorphous porous $\mathrm{Mn}_{3} \mathrm{O}_{4}$ negative electrode and $\mathrm{Ni}(\mathrm{OH})_{2}$ positive electrode: a novel and high-performance flexible electrochemical energy storage device. ACS Appl Mater Interfaces 7:11444-11451
19. Chen T, Peng H, Durstock M, Dai L (2014) High-performance transparent and stretchable all-solid supercapacitors based on highly aligned carbon nanotube sheets. Sci Rep 4:3612

20. Yao X, Luan C, Zhang D, Lan L, Fu J (2017) Evaluation of CF-embedded 3D printed structures for strengthening and structural-health monitoring. Mater Des 114:424-432

21. Chen Q, Li X, Zang X, Cao Y, He Y, Li P, Wang K, Wei J, Wu D, Zhu H (2014) Effect of different gel electrolytes on graphene-based solid-state supercapacitor. RSC Adv 4:36253-36256

22. MCDonald JC, Duffy DC, Anderson JR, Chiu DT, Wu H, Schueller OJA, Whitesides GM (2000) Fabrication of microfluidic systems in poly(dimethylsiloxane). Electrophoresis 21:27-40

23. Song B, Li L, Lin Z, Wu ZK, Moon K, Wong CP (2015) Water-dispersible graphene/polyaniline composites for flexible micro-supercapacitors with high energy densities. Nano Energy 16:470-478

24. El-Kady MF, Kaner RB (2013) Scalable fabrication of high-power graphene micro-supercapacitors for flexible and on-chip energy storage. Nat Commun 4:1475

25. Liu Z, Wu ZS, Yang S, Dong R, Feng X, Mullen K (2016) Ultraflexible In-Plan Micro-Supercapacitors by Direct Printing of Solution-Processable Electrochemically Exfoliated Graphene. Adv Mater 28:2217-2222

\section{Submit your manuscript to a SpringerOpen ${ }^{\odot}$ journal and benefit from:}

- Convenient online submission

- Rigorous peer review

- Open access: articles freely available online

- High visibility within the field

- Retaining the copyright to your article

Submit your next manuscript at $\gg$ springeropen.com 